\title{
An atomically quantized hierarchy of shear transformation zones in a metallic glass
}

\author{
J. D. Ju, ${ }^{1}$ D. Jang, ${ }^{2}$ A. Nwankpa, ${ }^{3}$ and M. Atzmon ${ }^{1,4, a)}$ \\ ${ }^{1}$ Department of Materials Science and Engineering, University of Michigan, Ann Arbor, Michigan 48109, USA \\ ${ }^{2}$ Department of Materials Science and Engineering, California Institute of Technology, Pasadena, California \\ 91125, USA \\ ${ }^{3}$ Computer Aided Engineering Network, University of Michigan, Ann Arbor, Michigan 48109, USA \\ ${ }^{4}$ Department of Nuclear Engineering and Radiological Sciences, University of Michigan, Ann Arbor, Michigan \\ 48109, USA
}

(Received 1 September 2010; accepted 24 December 2010; published online 15 March 2011)

\begin{abstract}
Quasistatic measurements of room-temperature anelastic relaxation were used to characterize the properties of shear transformation zones (STZs) in amorphous $\mathrm{Al}_{86.8} \mathrm{Ni}_{3.7} \mathrm{Y}_{9.5}$ in the dilute limit. Using a combination of nanoindenter cantilever bending and mandrel bend relaxation techniques, anelastic relaxation was measured over times ranging from $1 \mathrm{~s}$ to $3 \times 10^{7} \mathrm{~s}$. Direct spectrum analysis yields relaxation-time spectra, which display seven distinct peaks. The results were analyzed using a linear dashpot-and-spring model, combined with transition-state theory, to yield several STZ properties. These reveal a quantized hierarchy of STZs that differ from each other by one atomic volume. Potential STZs occupy a large volume fraction of the solid. They access their ergodic space, with the ratio of forward-to backward jump rates ranging from 1.03 to 4.3 for the range of stress values used. (C) 2011 American Institute of Physics. [doi:10.1063/1.3552300]
\end{abstract}

\section{INTRODUCTION AND BACKGROUND}

Metallic glasses exhibit high strength and elastic limit, properties that are attractive for a variety of structural applications. ${ }^{1}$ However, they also exhibit flow localization, which results in macroscopically brittle behavior. While significant progress has been made in understanding their mechanical behavior since the pioneering work by Spaepen, ${ }^{2}$ a detailed microscopic description of viscoelastic flow of metallic glasses remains a challenging task. Plastic deformation of crystalline materials has long been well-understood, and described in terms of well-defined lattice defects. However, glasses pose significant challenges in defining flow defects, as even the baseline structure is poorly known. Recent reviews of deformation of metallic glasses have been given by Schuh $e t a^{3}{ }^{3}$ and Trexler and Thadhani. ${ }^{4}$

Spaepen's model ${ }^{2}$ has been successful in describing a range of observations on flow and flow localization. Argon ${ }^{5,6}$ added microscopic details, based on insight gained from twodimensional bubble rafts. ${ }^{7,8} \mathrm{He}$ identified low-stress flow defects as microscopic, equiaxed, regions, termed shear transformation zones (STZs). The shear transformations are thermally activated and assisted by external stress, and the transformation shear strain, $\gamma_{0}^{T}$, is of the order of 0.1. Both authors expressed the shear rate in terms of transition-state theory, with a barrier height biased by an applied stress. Argon and Shi argued ${ }^{6}$ that isolated STZs can be reversed by back-stress in the elastic matrix, leading to macroscopic anelasticity. In fact, simulations ${ }^{9}$ and experiments ${ }^{10,11}$ show an anelastic contribution to apparent elastic behavior in metallic glasses, with the simulations showing a bond-breaking mech-

\footnotetext{
a) Author to whom correspondence should be addressed. Electronic mail: atzmon@umich.edu.
}

anism. Egami et al. ${ }^{12}$ correlated an elastic deformation with bond-orientational order. Argon and $\mathrm{Kuo}^{13}$ used temperature-stepping experiments to determine activation energy spectra for anelastic relaxation for several metallic glasses. Increased attention has recently been given to the behavior of shear transformation zones, using three-dimensional colloids ${ }^{14}$ and molecular dynamics (MD) simulations. ${ }^{15,16}$ Despite the progress made, the lack of matrix periodicity and the small size of STZs have made their direct experimental characterization elusive.

In order to investigate the properties of STZs, we have conducted quasistatic anelastic relaxation measurements in amorphous $\mathrm{Al}_{86.8} \mathrm{Ni}_{3.7} \mathrm{Y}_{9.5}$, an alloy previously studied by one of the authors. ${ }^{17,18}$ Unlike some Al-rich metallic glasses, this alloy does not crystallize upon room-temperature plastic deformation. It exhibits significant anelastic deformation at room temperature, enabling us to conduct stable, high resolution, measurements for durations of $1 \mathrm{~s}-3 \times 10^{7} \mathrm{~s}$. Our simple experiments provide valuable information on STZ properties. Most importantly, we obtain evidence of a quantized hierarchy of STZs with single-atom increments.

\section{EXPERIMENTAL PROCEDURE}

An amorphous $\mathrm{Al}_{86.8} \mathrm{Ni}_{3.7} \mathrm{Y}_{9.5}$ (at.\%) ribbon, $22 \mu \mathrm{m}$ thick and $1 \mathrm{~mm}$ wide, was obtained by the single-wheel melt-spinning technique using a $\mathrm{Cr}$-coated $\mathrm{Cu}$ wheel at a tangential velocity of $40 \mathrm{~m} / \mathrm{s}$ in vacuum. Electron diffraction analyses were employed to confirm the amorphous structure of the as-spun alloy ribbon.

All relaxation measurements were performed at $295 \pm 1$ K. An Agilent G200 nanoindenter with a DCM head was used for the cantilever measurements (Fig. 1(a)). Cantilever samples were mounted in epoxy for nanoindenter 


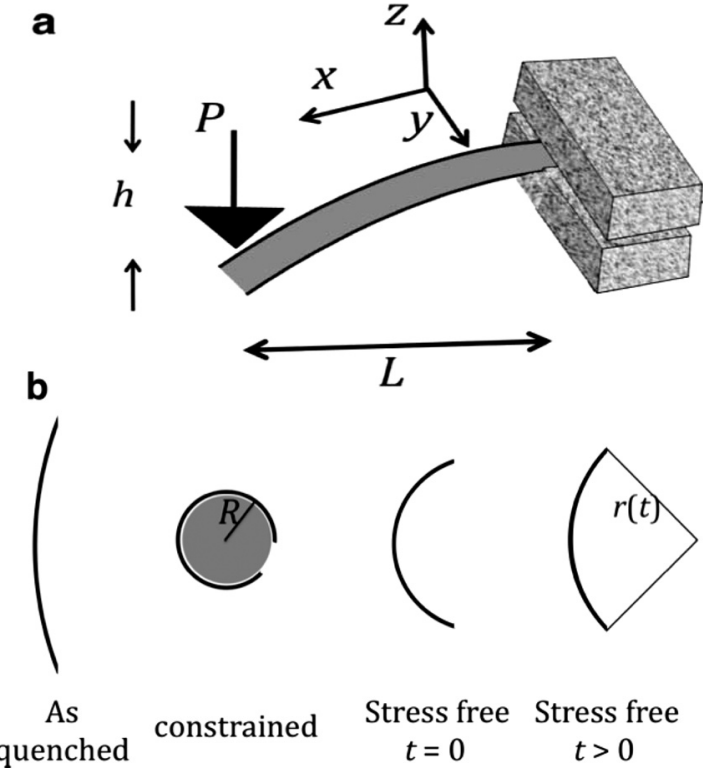

FIG. 1. Measurement techniques. (a) Cantilever method. The displacement $h$ is monitored as a function of time at a fixed load, $P$. The instantaneous displacement is the elastic component; (b) Mandrel method. The sample was constrained for $2 \times 10^{6} \mathrm{~s}$ at varying radii, after which the radius of curvature was monitored as a function of time in a stress-free condition.

experiments, and the distance between the clamp and indenter tip contact point was $0.1 \mathrm{~cm}$. Two different mounting compounds were used in order to rule out their effect on the measurement. Thermal drift was subtracted. In each run, the following cycle, consisting of three stages, was repeated 20 times: $0.2,0.02$, and $0.002 \mathrm{mN}$, each for $200 \mathrm{~s}$. The stiffness measured from the instantaneous, elastic, displacement agreed with the calculated value, ${ }^{19}$ based on a Young's modulus value of $E_{0}=48.2 \mathrm{GPa}$ (Ref. 20) for a similar alloy. Displacement versus time data obtained during each full-load stage were used in the analysis, each consisting of 5000 points. Reversibility was confirmed during the low-load parts of each cycle. Throughout this paper, $\varepsilon$ refers to the maximum bending $[x$ direction in Fig. 1(a)] strain, attained at the surface. For the cantilever, this maximum strain is attained at the fixed end of the sample, and is given by $\varepsilon=3 d \cdot h / 2 L^{2},{ }^{19}$ where $d$ is the sample thickness, $h$ its displacement, and $L$ its effective length. The elastic strain, $\varepsilon_{\mathrm{el}}^{0}$, is constant under fixed nanoindenter load, and is determined from the instant deflection in response to a jump in load. Its value is less than $10^{-4}$ for all measurements. The time-dependent strain value, normalized by $\varepsilon_{\mathrm{el}}^{0}$, is equal to the ratio of the corresponding displacements. A total of 95 measurement cycles were obtained from four different samples.

For the bend stress relaxation ("mandrel") experiment, ${ }^{21,22}$ Fig. 1(b), five $1 \mathrm{~cm}$ long samples were used. Samples were first constrained around mandrels of radii ranging from 0.35 to $0.49 \mathrm{~cm}$ for $2 \times 10^{6} \mathrm{~s}$, then allowed to relax stress-free for $3 \times 10^{7} \mathrm{~s}$. The radius of curvature was monitored during relaxation as a function of time using a digital camera, taking care to insure that its optical axis was perpendicular to the sample stage, which was illuminated with a backlight. The curvature was determined by on-screen visual fitting of the calibrated photo with a circle. Under constraint, the total bending strain at the surface is $\varepsilon^{T}=d / 2\left(1 / R-1 / r_{0}\right)$, where $R$ is the mandrel radius and $r_{0}$ is the initial sample radius of curvature. $\varepsilon^{T}$ consists of an elastic and anelastic contribution, the values of which at the end of the constraining period are $\varepsilon_{\mathrm{el}}^{0}=d / 2 \times[1 / R-1 / r(0)]$ and $\varepsilon_{\mathrm{an}}^{0}=d / 2 \times[1 /$ $\left.r(0)-1 / r_{0}\right]$, respectively, where $r(t)$ is the radius of curvature at time $t$ after removal of the constraint. Note that $\varepsilon_{\mathrm{el}}^{0}$, which is defined differently for the cantilever and mandrel experiment, is the elastic strain at mechanical equilibrium in both cases. The maximum bending strain at time $t$ after removal of the constraint is given by $\varepsilon(t)=d / 2 \times(1 / r(t)-1 /$ $\left.r_{0}\right)$. These expressions assume a neutral plane equidistant from the surfaces. Since we find the processes under consideration to be linear functions of the stress, the strain varies linearly across the sample thickness, justifying the assumption. For the different mandrel radii used, $\varepsilon_{\text {el }}^{0}$ ranged from $0.153 \%$ to $0.303 \%$. The maximum shear stress is given by $\sigma_{s}=\left(\sigma_{x}-\sigma_{z}\right) / 2=\sigma_{x} / 2=E_{0} \varepsilon_{x} / 2\left(1-v^{2}\right)$ since the out-ofplane stress, $\sigma_{z}$, is zero. The maximum shear strain is given by $\gamma=\varepsilon_{x}-\varepsilon_{z}=\varepsilon_{x} /(1-v)$, where $\varepsilon_{z}$ is the out-of-plane strain. It is important to note that because the in-plane perpendicular stress, $\sigma_{\mathrm{y}}$, is proportional to $\sigma_{\mathrm{x}}$, relative changes in $\sigma_{\mathrm{x}}$ and $\varepsilon_{\mathrm{x}}$ due to any linear process are the same as they would be for uniaxial geometry.

Direct spectrum analysis ${ }^{23}$ was performed by fitting the relaxation curves, using the Primal-Dual Interior Point Filter Line Search Algorithm. The software package AMPL (Ref. 24) was used with nonlinear solver IPOPT. ${ }^{25}$ In the fits, the relaxation-time values, $\tau_{\mathrm{i}}$, were fixed and spaced logarithmically in the ranges 0.3 to $400 \mathrm{~s}$ and 1080 to $5.4 \times 10^{7} \mathrm{~s}$ for the cantilever and mandrel experiments, respectively. Each experimental curve was fitted to obtain a relaxation-time spectrum, $f(\tau)$. To obtain the integral over peak $m, \int_{m} f(\tau) d \ln \tau$, for overlapping peaks, $f(\tau) / \tau$ was fitted with sums of log-normal functions, yielding excellent agreement. STZ properties were calculated for each peak in each spectrum, and then averaged over the spectra. The error indicated in the plots is the standard deviation of the mean.

\section{RESULTS AND DISCUSSION}

The maximum anelastic bending strain of mandrel samples, equilibrated under constraint for $2 \times 10^{6} \mathrm{~s}$ and subsequently allowed to recover, is shown, normalized by $\varepsilon_{\mathrm{el}}^{0}$, as a function of time in Fig. 2. As with any static measurement, $\varepsilon_{\mathrm{el}}^{0}$ may include anelastic contributions ${ }^{10,11}$ with time scales shorter than the experimental resolution. The curves in Fig. 2 indicate absence of significant permanent strain and are independent of $\varepsilon_{\mathrm{el}}^{0}$. The implied linearity in stress leads to important conclusions: a) the anelasticity is unlikely to originate from macroscopically heterogeneous behavior, but rather has microscopic origin, b) the anelastic sites are not exhausted for the strain values used, c) the viscosity in the linear solid model, used below, is Newtonian, i.e., the strain rate of the dashpots is proportional to their stress, and d) consequently, unlike for the case of yield, the strain profile across the sample thickness is linear at any time. In Fig. 2, for the highest value of applied strain, the maximum anelastic bending strain at the end of the constraining period, $t=0$, is about $0.055 \%$. Assuming the shear strain of an STZ (Ref. 5) when 


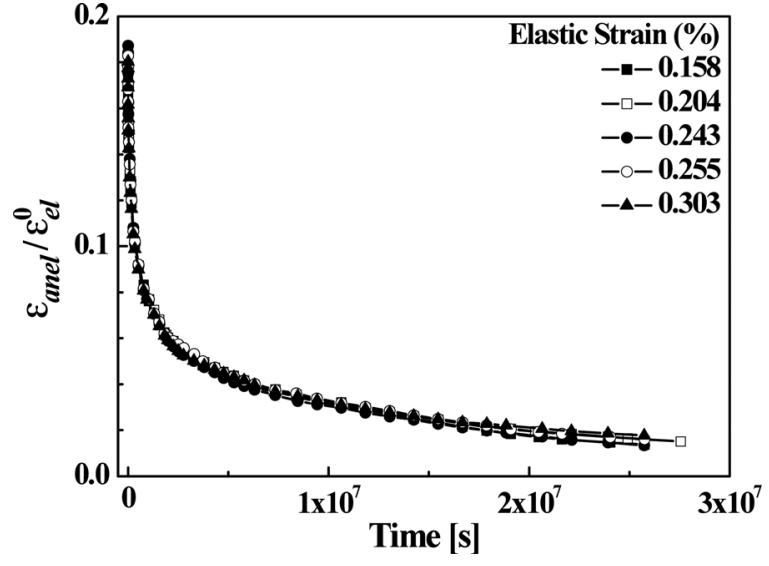

FIG. 2. Anelastic strain evolution following equilibration at different mandrel radii. The strain is normalized by the elastic strain at equilibrium, prior to removal of the constraint.

constrained by the surrounding matrix is $\gamma_{0}^{c}=0.09$ (see below), and converting bending to shear strain, the corresponding total volume fraction occupied by activated STZs is about $0.94 \%$.

In order to observe anelastic relaxation with time scales of 1-200 s, the displacement of cantilever samples at constant load, $P=0.2 \mathrm{mN}$, Fig. 1(a), was monitored as a function of time. Its division by the instantaneous displacement upon loading yielded the ratio of anelastic to elastic bending strain, $\varepsilon_{\mathrm{an}}(t) / \varepsilon_{\mathrm{el}}^{0}$. Sample curves for both measurements are shown in Figs. 3(a) and 3(b).

Because anelastic relaxation in a metallic glass involves several processes, the temporal evolution of the strain is typically fitted with a linear combination of exponentially decaying terms, $\exp (-t / \tau)$, with different time constants, $\tau$. Alternatively, a single stretched exponent has been used, $\exp \left(-(t / \tau)^{\beta}\right),{ }^{26}$ implicitly making an assumption about the shape of the relaxation spectrum. Instead, we employ a direct spectrum analysis method ${ }^{23}$ by fitting the following functions to the anelastic strain as a function of time:

$$
\begin{gathered}
\varepsilon=A+B t+\sum_{i=1}^{N_{1}} \varepsilon_{i} \exp \left(-t / \tau_{i}\right), \\
\varepsilon=A+\sum_{i=1}^{N_{2}} \varepsilon_{i} \exp \left(-t / \tau_{i}\right)
\end{gathered}
$$

for the cantilever and mandrel measurement, respectively, with $N_{1}$ and $N_{2}$ less than the number of data points. The $\varepsilon_{i}$ are fitting parameters, and the $\tau_{\mathrm{i}}$ are fixed and spaced logarithmically. The linear term in Eq. (1a) a priori describes plastic flow or approximates anelastic processes with time constants greater than the measurement duration. $A$ in Eq. (1b) is very small and likely corresponds to processes with time constants longer than the duration of the experiment.

It should be noted that even in a simulated relaxation curve consisting of a sum of a small number of pure exponential terms that differ from each other by a factor of ten, it is difficult to discern the different time constants. This is especially true when the amplitude of the exponents with the longest time constants is the greatest, since these introduce a curvature in a $\log (\varepsilon)$ versus time plot at short times. Experimental noise, and the fact that the spectrum peaks have an intrinsic width, further obscure any distinct processes. Because of the consequent need to rely on fits, we undertook several steps to rule out artifacts due to the fitting method. We have: a) performed fits with several values of $N_{1}, N_{2}$; and b) fitted the mandrel data with 4-8 exponents, allowing both the $\varepsilon_{i}$ and $\tau_{i}$ to vary. The results of the different methods were consistent with each other. Regardless of the number of exponents used, the same dominant time constants were obtained. In addition, simulated data, containing noise, were fitted in order to assess the method's reliability. While the peak widths varied with initial guesses, their areas did not.

The spectra resulting from the fits, $f(\tau)$, are included in Figs. 3(a) and 3(b). These are normalized such that $\varepsilon_{\mathrm{el}}^{0} \times \int_{\tau_{1}}^{\tau_{2}} f(\tau) d \ln \tau$ is the equilibrium anelastic bending strain due to processes with time constants in any interval $\left(\tau_{1}, \tau_{2}\right) . f(\tau) / \tau$ exhibits a distinct set of peaks, each described well with a log-normal distribution.

We associate each peak in the spectra with one STZ type, $m$. To analyze the relaxation behavior, the standard linear solid model ${ }^{27,28}$ is used, as illustrated in Fig. 4: a Voigt unit $m$, consisting of a spring and linear dashpot in parallel, represents all STZs of type $m$, corresponding to one peak in the spectrum [Figs. 3(a) and 3(b)]. Since the strain contribution of
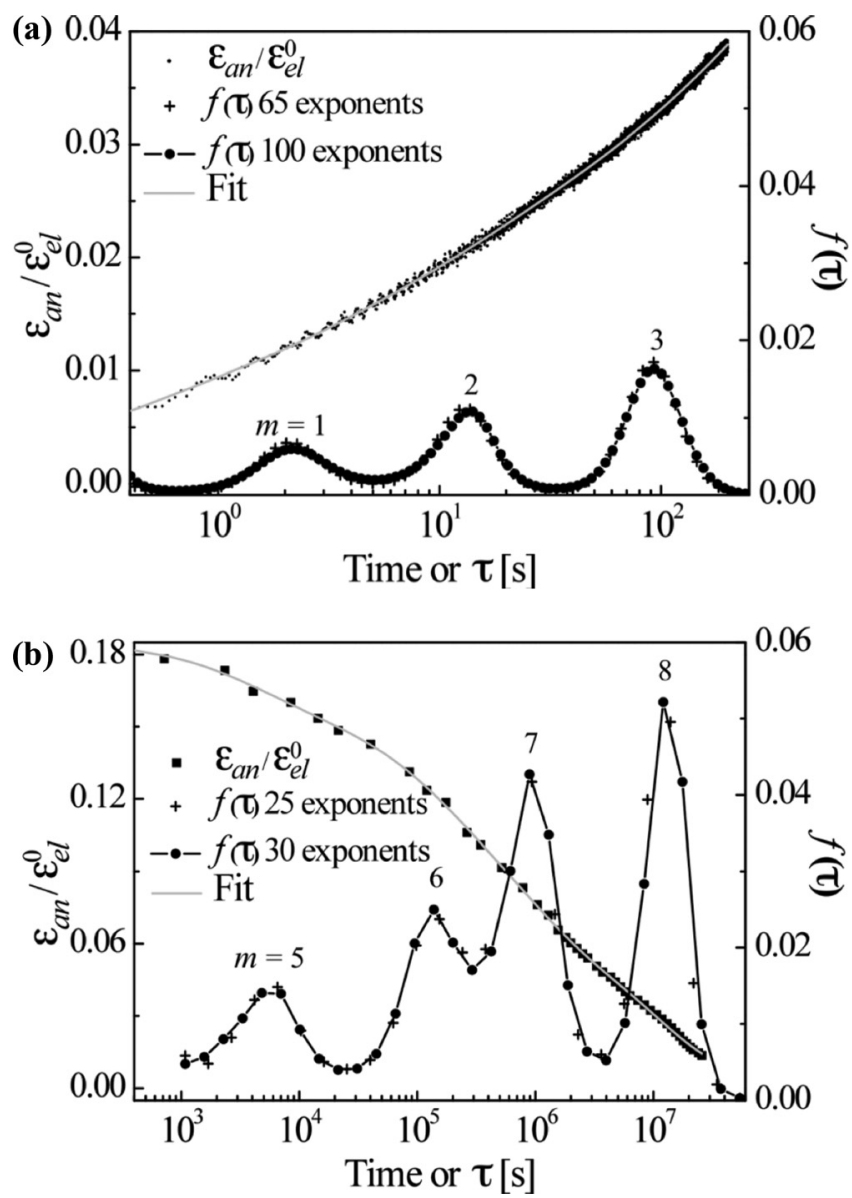

FIG. 3. Sample relaxation curves and corresponding relaxation-time spectra. (a) Cantilever measurement, performed at fixed load, $P=0.2 \mathrm{mN}$, i.e., fixed stress. (b) Mandrel measurement, performed in a stress-free condition after equilibration under constraint. For each case, two spectra, $f(\tau)$, are shown, obtained from fits with different numbers of fitting parameters (see text). 


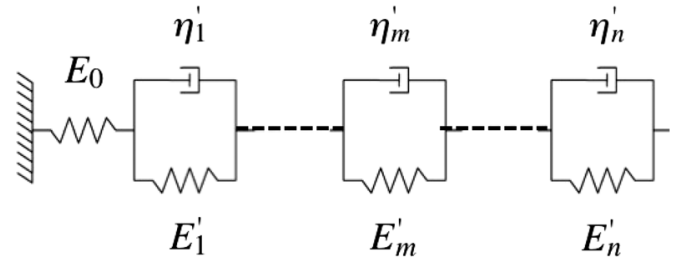

FIG. 4. Linear solid model: $n$ anelastic processes act in series, where $m$-type sites are associated with Young's modulus of $E_{m}^{\prime}$ and viscosity $\eta_{m}^{\prime}$, both effective quantities that are inversely proportional to the volume fraction of these sites.

each STZ type is additive, the units are connected in series. The additivity implies that both the effective Young's modulus and shear viscosity, $E_{m}^{\prime}$ and $\eta_{m}^{\prime}$, respectively, are inversely proportional to the density of $m$-type STZs. In the limit of vanishing concentration of $m$-type STZs, $E_{m}^{\prime}, \eta_{m}^{\prime} \rightarrow \infty$, i.e., the corresponding Voigt unit is rigid and makes a negligible contribution to the strain. It is important to recognize the limitations of this simple model: Argon and $\mathrm{Shi}^{6}$ note that once an STZ has other STZs as neighbors, its transformation is likely to become irreversible. This is equivalent to destruction of the spring that is parallel to a dashpot. In the present work, conducted at low strains and showing full reversibility, such a process is not encountered.

Using appropriate boundary conditions, exponential relaxation of stress or strain is obtained. Under fixed or zero stress, the strain in each unit evolves independently, with time constant,

$$
\tau_{m}=\frac{3 \eta_{m}^{\prime}}{E_{m}^{\prime}},
$$

where the factor of 3 accounts for the conversion of uniaxial to shear viscosity. $\tau_{m}$ will be taken as the median of the respective (log-normal) peak, since it will yield the peakaveraged activation energy, $\Delta F_{m}$, below.

Mechanical equilibrium between unit $m$ and the spring in Fig. 4 yields the effective Young's modulus associated with the unit:

$$
E_{m}^{\prime}=\frac{\varepsilon_{\mathrm{el}}^{0}}{\varepsilon_{m}^{0}} E_{0},
$$

where $E_{0}$ is Young's modulus, and $\varepsilon_{m}^{0}=\varepsilon_{\mathrm{el}}^{0} \times \int_{m} f(\tau) d \ln \tau$, with integration over peak $m$, is the anelastic bending strain due to $m$-type sites at mechanical equilibrium. Eq. (3) would remain the same if the ratio of shear strains were used, since these are linear in the corresponding bending strains for the present geometry. The assumption of mechanical equilibrium between each STZ type and the matrix is valid for all but $m=8$, since $\tau_{8}=1.25 \times 10^{7} \mathrm{~s}$, as compared with a constraint duration of $2 \times 10^{6} \mathrm{~s}$. A corresponding correction is implemented in the analysis below.

Once $E_{m}^{\prime}$ and $\tau_{m}$ are determined from the spectra, Eq. (2) is used to calculate $\eta_{m}^{\prime}$. Below, we will relate it to the additive contribution of $m$-type STZs to the macroscopic anelastic shear strain rate,

$$
\dot{\gamma}_{m}=\frac{\sigma_{s}}{\eta_{m}^{\prime}},
$$

where $\sigma_{\mathrm{s}}$ is the net shear stress on the dashpot in Voigt unit $m$, equal to the applied stress minus that of the corresponding spring. Since our samples exhibit linear deformation behavior, $\eta_{m}^{\prime}$ is constant, and $\sigma_{s}$ and $\dot{\gamma}_{m}$ vary linearly, across the thickness. Therefore, Eq. (4) is valid for the entire sample. Following our convention for $\varepsilon$, maximum values of $\sigma_{\mathrm{s}}$ and $\dot{\gamma}_{m}$, attained at the surface, are used in the analysis below.

The total volume of potential, (also known as fertile), $m$-type STZs, per unit volume can be expressed as

$$
c_{m}=\frac{\varepsilon_{m}^{0}}{\varepsilon_{\mathrm{el}}^{0}}=\frac{E_{0}}{E_{m}^{\prime}},
$$

as derived in the Appendix. The full physical meaning of $c_{m}$ is discussed below. Equation (5) is obtained by modeling an anelastic unit $m$ as a large number of potential STZs in series, all of which are reversible due to the same elastic constant as the solid. ${ }^{2,5}$ The main step in modeling STZs, which have on/off states, with Voigt units that have a continuum of strain states is based on the ergodicity of STZs: since equilibrated STZs undergo thermal fluctuations that are only biased by the stress (see below and Ref. 14), the ensemble average, the activated fraction, $x_{m}$, of $c_{m}$ at mechanical equilibrium, can be interpreted as the average fraction of time each potential STZ is transformed. Thus, an STZ has a continuum of time-averaged strain values. While kinetic measurements can involve significant uncertainty in preexponential factors, Eq. (5) allows for a reliable determination of $c_{m}$ from experiment, which does not depend on $\gamma_{0}^{T}$ and applies to any STZ type that has equilibrated.

Using the literature value, $E_{0}=48.2 \mathrm{GPa}$ for a similar alloy, ${ }^{20} \tau_{m}, c_{m}, E_{m}^{\prime}$, and $\eta_{m}^{\prime}$ were calculated for each $m$ by averaging over values obtained from the individual spectra, Fig. 5(a)-5(d). For $m=4$, only $\eta$ is given, as determined from the linear part of the nanoindenter curve. It likely corresponds to an anelastic process with $\tau$ values that fall between the ranges covered by the two measurement methods. We observe $c_{m}$ to range from $<1 \%$ for the fastest sites to $\sim 27 \%$ for the slowest: the total volume fraction occupied by potential STZs is a significant fraction of unity. In this context, we point out that we view $c_{m}$ as the total volume of potential type- $m$ STZs per unit volume, where overlapping volumes are counted multiple times. While our analysis is restricted to $x_{m} \ll 1$, i.e., a small volume fraction of the potential STZs are activated, $c_{m}$ values greater than 1 are meaningful: the contribution of two potential STZs that have a finite spatial intersection to the probability of STZ formation is proportional to the sum of their volumes as long as their activation is rare. $c_{m}>1$ merely implies that the anelastic strain is greater than the elastic strain [Eq. (5)]. For the present experimental conditions, at any point in time, the majority of potential STZs are not activated and are part of the elastic matrix. Thus, a high $c_{m}$ value does not affect $E_{O}$ in Fig. 4 if $x_{m} \ll 1$. It should be noted that while $c_{m}$ may continue to increase with increasing $m$, STZs with high $m$ will be kinetically frozen below the glass transition temperature [Eq. (7)].

After an anelastic site type with time constant $\tau$ is activated at fixed stress for a duration $t_{0} \ll \tau$, the stress-free 

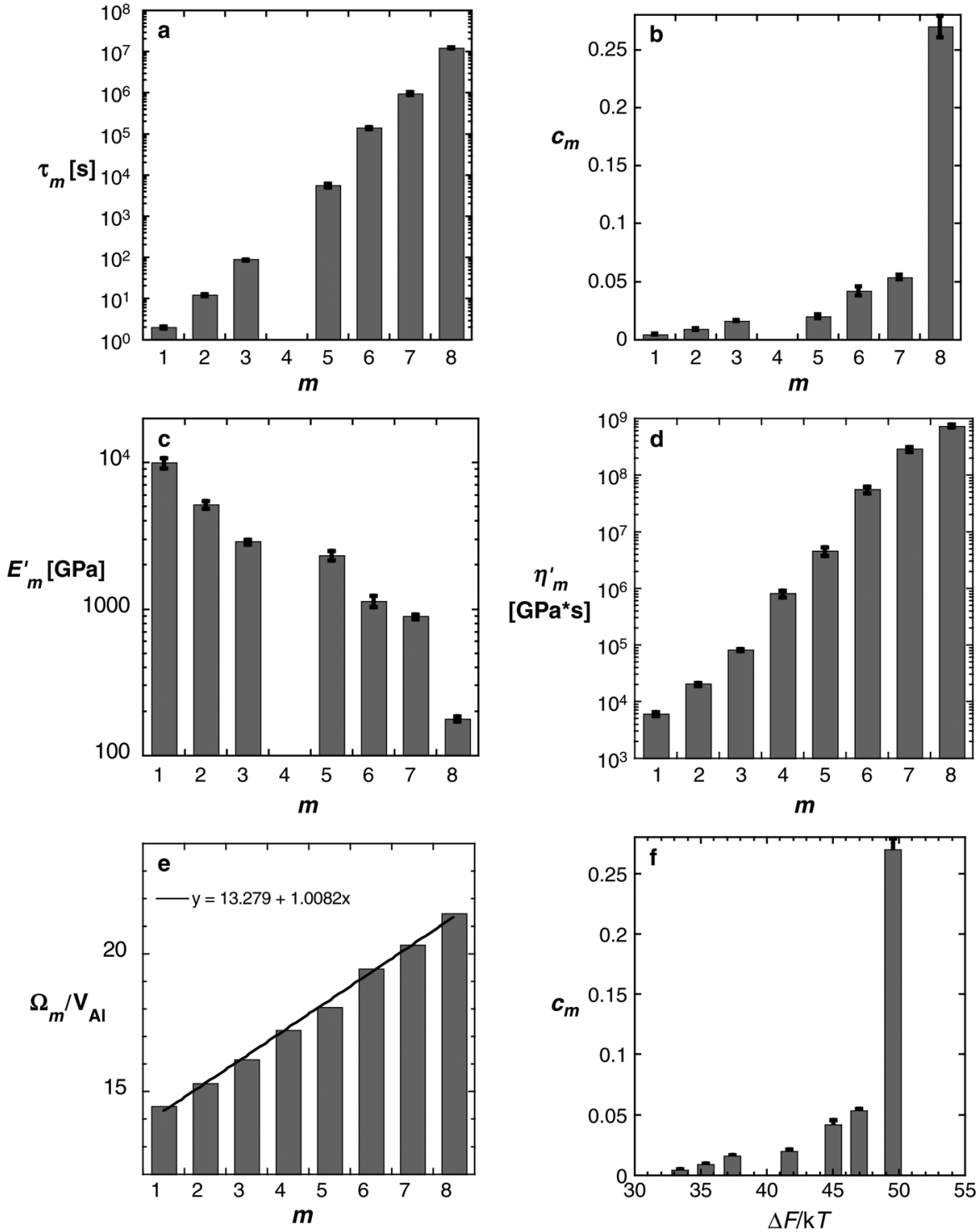

FIG. 5. Calculated properties of the respective STZ types $m=1-8$. (a) Time constants. (b) Volume fraction of potential STZs; (c) Effective macroscopic Young's modulus. (d) Effective macroscopic viscosity. (e) STZ volume in units of atomic volume of $\mathrm{Al}, \mathrm{V}_{\mathrm{Al}}=16.6 \times 10^{-30} \mathrm{~m}^{3}$. The value for $m=4$ was obtained from Fig. 4(d) and interpolation in Fig. 4(b). (f) Volume fraction of potential STZ and transformation strain as a function of $\Delta F / k T$. The error bars are the standard deviation of the mean, obtained by averaging over multiple measurements. strain relaxation rate is smaller than that under stress by a factor of $t_{0} / \tau$. Therefore, anelastic strains may last significantly longer than the duration of the prior stress that caused them, a fact that is often missed. This is the case for $m=8$ in the present work. The reader is reminded that the present value of $c_{8}$ is determined by extrapolation to account for the fact that equilibrium is not reached for $m=8$ during the constraining period. The extrapolation introduces added error, which will be reduced in our ongoing work.

We proceed to determine the STZ volume values. Previous derivations of the shear strain rate were based on one dominant STZ type. ${ }^{2,5,6}$ Since our experiments resolve different STZ types, we follow those derivations to express the contribution to the total shear strain rate due to STZs of type $m$ :

$$
\dot{\gamma}_{m}=2 c_{m} \gamma_{0}^{c} v_{\mathrm{G}} \exp \left(-\frac{\Delta F_{m}}{k T}\right) \sinh \left(\frac{\sigma_{s} \gamma_{0}^{T} \Omega_{m}}{2 k T}\right)
$$

where $\Omega_{m}$ is the STZ volume and $\gamma_{0}^{c}=[2(4-5 v) /$ $15(1-v)] \gamma_{0}^{T}$ is Poisson's ratio, $v_{\mathrm{G}}$, the attempt frequency, and $k T$ has its usual meaning. The activation free energy for an $m$-type STZ is:

$$
\Delta F_{m}=\left[\left(\frac{(7-5 v)}{30(1-v)}+\frac{2(1+v)}{9(1-v)} \bar{\beta}^{2}\right) \gamma_{0}^{T}+\frac{1}{2} \frac{\bar{\sigma}_{\mathrm{STZ}}}{\mu}\right] \mu \gamma_{0}^{T} \Omega_{m},
$$

where $\bar{\beta}^{2} \sim 1$ the dilatancy factor, $\mu=E_{0} /[2(1+v)]$, the shear modulus and $\bar{\sigma}_{\mathrm{STZ}}$ the shear resistance of the STZ. The division of the mechanical work term in Eq. (6) by two reflects the assumption that the mechanical energy is a linear function of strain between the two STZ configurations ${ }^{2}-$ there are other models that also satisfy detailed balance. The other factor of two, absent in Refs. 5 and 6, originates from the subtraction of forward- and backward flux and the definition of the sinh function. The following values were assumed for all STZ sizes: $v_{\mathrm{G}}=10^{13} \mathrm{~s}^{-1}, v=0.35, \bar{\sigma}_{S} / \mu=0.025$ (Ref. 29) and $\gamma_{0}^{T}=0.2$. In Ref. $6, \gamma_{0}^{T}$ values range from 0.1 to 0.135 , with a different mechanical work term. Colloid experiments yield $\sim 0.3 .^{14,30}$ It is noted that the empirical definition of the activation volume, $V^{*}=k T d \ln \dot{\gamma} / d \sigma$, 
equals $\gamma_{0}^{T} \Omega / 2$ only if $\sigma_{s} \gamma_{0}^{T} \Omega / 2 k T \gg 1$. Caution should be exercised when assigning a physical meaning to $V^{*}$ in other regimes. Using Eq. (6), linearized for small $\sigma_{\mathrm{s}}$, and Eqs. (4) and (7), $\Omega_{m}$ is obtained. Its value, normalized by the atomic volume of $\mathrm{Al}$, is displayed in Fig. 5(e). A hierarchy of $\Omega_{m}$ values is observed, ranging from $\sim 14$ to $\sim 21$, in singleatomic increments.

$\Omega_{m}$ is insensitive to error in $\eta_{m}^{\prime}$ since it appears in the exponent. It is, obviously, dependent on the assumed value of $\gamma_{0}^{T}$. Despite the uncertainty in the latter, the magnitude of the $\Omega$ spacings strongly suggests that the peaks in the relaxation spectrum correspond to a quantized hierarchy of STZs with single-atomic increments. Since the local chemistry and structure in an amorphous solid are expected to have a wide distribution, the clear separation of the processes may be surprising. Based on detailed studies of two dimensional bubble-rafts, Argon ${ }^{5,8}$ argued that the spectrum of activation energies reflects the expected free-volume distribution: a large volume fraction occupied by sites with small free volume, and therefore a high activation barrier, and vice versa. In light of our present experiments, it appears that the spectrum of activation energies, calculated from Eq. (7) and displayed in Fig. 5(f), reflects the discrete STZ sizes and not the free-volume distribution. Spatial fluctuations in the composition, density and elastic constants are averaged over a volume that includes the surrounding matrix, and are apparently insufficient to obscure the effect of discrete STZ volumes. This argument is consistent with the fact that the third term in Eq. (7), the work required to shear the atomic planes in an STZ, is insignificant, ${ }^{6}$ and with MD simulations ${ }^{15}$ that show a well-defined composition, and narrow distribution of the volume per atom, in activated STZs. We suggest that the local state of structural relaxation, i.e., the distribution of free volume or stress fluctuations, affects the flow behavior as an on/off switch via $c_{m}$. Our ongoing work is expected to lead to further insight into this issue.

Our analysis implies that STZs that are larger or smaller than those detected should be active for the appropriate temperature and time scale. While extrapolation suggests that smaller STZs makeup a small volume fraction, it also suggests steeply increasing volume fractions occupied by STZs with increasing $m$. Previous publications have all reported single, average, STZ sizes. Because of the steep increase of $c_{m}$ with $m$ [Fig. 5(b)], it is expected that the contribution of the largest active STZs will dominate the macroscopic strain. Based on Eq. (6), it follows that the observed average STZ size will increase with increasing temperature. In the present work, the volumes of the different STZs that contribute to the observed relaxation range from 14 to 21 atomic volumes of $\mathrm{Al}$, in single-atom increments, as compared with 53 atomic volumes for the slowest, and therefore largest, active STZs determined for $\mathrm{Pd}_{80} \mathrm{Si}_{20}$ at elevated temperature. ${ }^{6}$ Considering our uncertainty in $\gamma_{0}^{T}$, these results are not inconsistent with the present work. Pan et al., ${ }^{31,32}$ using strain-rate sensitivity measurements by nanoindentation, reported STZ sizes as high as $>680$ atoms. However, such measurements involve strain localization and shear band formation. The deforming volume fraction increases with increasing strain rate, ${ }^{33,34}$ which leads to an underestimate of the microscopic strain rate sensitivity and therefore an overestimate of the STZ volume. In addition, the state of relaxation affects pileup ${ }^{18}$ and therefore the indenter contact area. Therefore, we do not consider the high $\Omega$ values in Refs. 31 and 32 to be realistic. MD results show STZs consisting of $2-10$ atoms $^{15}$ and tens to hundreds of atoms, increasing with strain. ${ }^{16}$ Such simulations are conducted at higher stress and strain than the present experiment, and caution should be exercised when using them for comparison, as they are likely to be affected by interaction among STZs. It should also be noted that thermally activated shear of STZs well below the glass transition temperature is a rare event, which cannot be modeled realistically by MD.

Dmowski et al. ${ }^{11}$ have recently concluded from measured anisotropic atomic pair distribution functions that $1 / 4$ of the volume of a Zr-based metallic glass deforms anelastically with short time constants, contributing to the apparent elastic behavior on typical experimental time scales. If we extrapolate our $c_{m}$ values to smaller $m$, and therefore shorter $t$, we obtain very small volume fractions. This discrepancy is even greater than it appears, because the alloy of Ref. 11 has a higher glass transition temperature than the present alloy and its STZs should be more sluggish at room temperature for a comparable size. We suggest that the fast anelastic sites reported to occupy $1 / 4$ of the volume are of a different nature than the STZs that are active at high temperature, even though their effect on the pair distribution function may be similar. Activity of these sites is possibly described by a bond-exhange ${ }^{9}$ mechanism. Recent dynamic measurements in several metallic glasses are consistent with this picture, showing a nonzero loss modulus at cryogenic temperatures, which is separate from the broad high-temperature peak. ${ }^{35}$

Our $\Omega$ values are consistent with the assumption of Newtonian viscosity-for the highest stress values used, linearization of the sinh term in Eq. (6) results in an error of 6.5 to $9 \%$ for $\Omega_{5}$ to $\Omega_{8}$, and orders of magnitude less for the cantilever experiment. As in Ref. 14, STZ activation is thermal, with the stress bias resulting in an initial ratio of forward-tobackward jump rates of $\sim 1.03$ for the cantilever and 3.4-4.3 for the mandrel experiment. As seen in Fig. 5(f), $\Delta F_{m} / k T$ ranges from about 33 to $50.0 .85<\Delta F_{m}<1.26 \mathrm{eV} /$ atom, compared with a vacancy migration energy in crystalline $\mathrm{Al}$, $\Delta H=0.62 \mathrm{eV}^{36}$ The present experiment is conceptually related to the measurement of the activation-energy distribution by temperature stepping, ${ }^{13}$ but it does not suffer from resolution limitations due to thermal stabilization times. Reference 13 yields $0.87<\Delta F<2.18 \mathrm{eV} /$ atom for alloys with shear moduli in a range above twice that of the present alloy, which represents good agreement. It is important to note that our $\Omega$ values are dependent on the assumed value of $\gamma_{0}^{T}$ - using 0.3 would reduce $\Omega$ by a factor greater than two, whereas $\gamma_{0}^{T}=0.1$ would increase it by a factor of approximately 4 . Our assumption of size-independent $\gamma_{0}^{T}$ is unlikely to significantly affect our conclusions.

Anelastic relaxation spectra with distinct peaks have been observed for other metallic glass alloys, ${ }^{37-40}$ indicating that our results are far from unique to the present alloy. In addition to the quantitative information we obtain, a novel 
aspect of the present work is the wide range of accessible time constants, which has allowed us to observe an unprecedented number of distinct processes. Few experiments, and no MD simulation, can access such a wide dynamic range. It may be surprising that no distinct anelastic processes were observed in any of the large number of dynamic internal-friction studies ${ }^{41-45}$ performed in metallic glasses over the years, since these are capable of exploring a wide dynamic range of time constants. Two factors obscure spectrum details in dynamic measurements, and are the likely cause: a) The anelastic response curve in dynamic internal friction measurements has a Cauchy frequency dependence, which leads to significant overlap among different processes even if they have low intrinsic width and differ from each other by a factor of 10. In contrast, and unlike most instrumented methods, the curvature measurements we conduct provide high resolution and stability over long time periods. Furthermore, b) dynamic measurements require high frequencies and therefore elevated temperatures. Since the time constant for a relaxation process is proportional to $\exp (\Delta F / \mathrm{k} T)$, a higher temperature will reduce the dynamic range of time constants and thus the resolution. We conclude that while our experimental approach is time consuming, it has been crucial to our discovery of the quantized hierarchy of STZs. Finally, we point out the difference between our regime of low strain and that of a highly driven flow state, for which simulations ${ }^{46}$ yield a broad and continuous distribution of activation energies.

In summary, quasistatic measurements of anelastic relaxation in $\mathrm{Al}_{86.8} \mathrm{Ni}_{3.7} \mathrm{Y}_{9.5}$ have yielded a range of $\mathrm{STZ}$ properties. A quantized hierarchy of STZs is revealed, with increments of a single atomic volume. The volume fraction occupied by potential STZs is obtained directly, and shown to be a large fraction of unity. While only a small fraction of the STZs is activated at any time for the low strains used, thermal fluctuations cause all potential STZs to probe their ergodic space and therefore have the same time-averaged strain. Future work will address activation of slower processes, dynamic measurements of possible anelastic processes that appear as elastic at our time resolution, the temperature dependence of anelastic relaxation and the effect of structural relaxation.

\section{ACKNOWLEDGMENTS}

This work was funded by the US National Science Foundation (NSF), Grant No. DMR-0605911. The authors are grateful to Professor F. Spaepen for useful discussions and reading of this manuscript, and T. Egami for useful discussions. They also thank Dr. F. Pinkerton of General Motors R\&D Center for preparing the sample used in this work, and Professor J. Greer for the use of the nanoindenter in her laboratory.

\section{APPENDIX: DERIVATION OF THE VOLUME FRACTION OCCUPIED BY POTENTIAL STZS}

The connection between the standard linear solid model (Fig. 4), with a continuum of strain states, and STZs, which have discrete strain states, is developed here. As illustrated

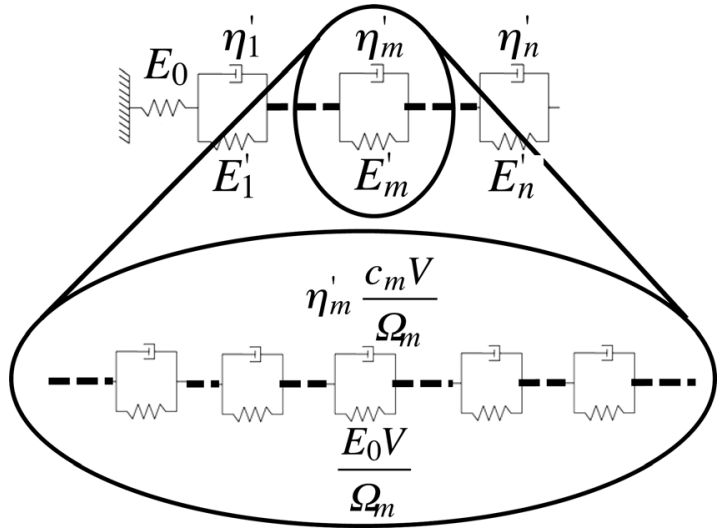

FIG. 6. The linear model used for individual $m$-type STZs. Voigt unit $m$ in Fig. 4 consists of many Voigt units, each representing a single STZ, in series. The effective viscosity and effective Young's modulus of each STZ is inversely proportional to the volume fraction it occupies. $V$ is the volume of the solid.

in Fig. 6, Voigt unit $m$ in Fig. 4 is modeled as a large number of Voigt units in series, each representing the additive contribution of one $m$-type STZ to the strain. The macroscopic shear strain due to $m$-type STZs is proportional to the fraction, $x_{m}$, of potential $m$-type STZs that are activated, and is therefore given by $\gamma_{m}=c_{m} x_{m} \gamma_{0}{ }^{c} \cdot \gamma_{0}{ }^{c}$ is the transformation strain of an STZ that is constrained by the elastic matrix. The macroscopic bending strain corresponding to $\gamma_{m}$ is

$$
\varepsilon_{m}=(1-v) c_{m} x_{m} \gamma_{0}^{c}
$$

where $v$ is Poisson's ratio.

$x_{m} \gamma_{0}^{c}$ is the ensemble-averaged shear strain of all potential STZs. Since the STZs are ergodic (see text and Ref. 14), $x_{m} \gamma_{0}^{c}$ is also equal to the time-averaged shear strain of each potential STZ. In this interpretation, all STZs participate in the deformation process and have a continuum of possible time-averaged strain values that evolve under a macroscopic applied stress. When an STZ is not in an activated state, it is part of the elastic matrix. Since the elastic constant that governs STZ reversal is the same as that of the matrix, the timeaveraged equilibrium strain of each STZ in the bending direction, $(1-v) x_{m}^{0} \gamma_{0}^{c}$, is equal to $\varepsilon_{\mathrm{el}}^{0}$, where $x_{m}^{0}$ is the value of $x_{m}$ at mechanical equilibrium. Applying Eq. (A1) at mechanical equilibrium, $\varepsilon_{m}^{0}=(1-v) c_{m} x_{m}^{0} \gamma_{0}^{c}$, in combination with Eq. (3), the condition for mechanical equilibrium, one obtains

$$
c_{m}=\frac{\varepsilon_{m}^{0}}{\varepsilon_{\mathrm{el}}^{0}}=\frac{E_{0}}{E_{m}^{\prime}} .
$$

As discussed in the text, $c_{m}$ can be understood as the total STZ volume per unit volume, with STZ intersections counted multiple times.

\footnotetext{
${ }^{1}$ A. Inoue, B. Shen, and A. Takeuchi, Mater. Sci. Forum 539-543, 92 (2007).

${ }^{2}$ F. Spaepen, Acta Metall. 25, 407 (1977).

${ }^{3}$ C. A. Schuh, T. Hufnagel, and U. Ramamurty, Acta Mater. 55, 4067 (2007).

${ }^{4}$ M. M. Trexler and N. N. Thadhani, Prog. Mater. Sci. 55, 759 (2010).

${ }^{5}$ A. S. Argon, Acta Metall. 27, 47 (1979).

${ }^{6}$ A. S. Argon and L. T. Shi, Acta Metall. 31, 499 (1983).
} 
${ }^{7}$ A. S. Argon and H. Y. Kuo, Mater. Sci. Eng. 39, 101 (1979).

${ }^{8}$ A. S. Argon and L. T. Shi, Phil. Mag. A 46, 275 (1982).

${ }^{9}$ Y. Suzuki and T. Egami, J. Non-Cryst. Sol. 75, 361 (1985).

${ }^{10}$ J. C. Ye, J. Lu, C. T. Liu, Q. Wang, and Y. Yang, Nature Mater. 9, 619 (2010).

${ }^{11}$ W. Dmowski, T. Iwashita, C. P. Chuang, J. Almer, and T. Egami, Phys. Rev. Lett. 105, 205502 (2010).

${ }^{12}$ T. Egami, W. Dmowski, P. Kosmetatos, M. Boord, T. Tomida, E. Oikawa, and A. Inoue, J. Non-Cryst. Solids 192-193, 591 (1995).

${ }^{13}$ A. S. Argon and H. Y. Kuo, J. Non-Cryst. Sol. 37, 241 (1980).

${ }^{14}$ P. Schall, D. A. Weitz, and F. Spaepen, Science 318, 1895 (2007).

${ }^{15}$ F. Delogu, Phys. Rev. Lett. 100, 255901 (2008).

${ }^{16}$ M. Neudecker and S. G. Mayr, Acta Mater. 57, 1437 (2009).

${ }^{17}$ W. H. Jiang and M. Atzmon, J. Mater. Res. 20, 696 (2005).

${ }^{18}$ W. H. Jiang, F. E. Pinkerton, and M. Atzmon, Acta Mater. 53, 3469 (2005).

${ }^{19}$ T. P. Weihs, S. Hong, J. C. Bravman, and W. D. Nix, J. Mater. Res. 3, 931 (1988).

${ }^{20}$ J. M. Freitag, R. G. Koknaev, R. Sabet-Sarghi, M. Koknaeva, and Z. Altounian, J. Appl. Phys. 79, 3967 (1996).

${ }^{21}$ J. Haimovich and T. Egami, Mater. Sci. Eng. 61, 89 (1983).

${ }^{22}$ C. Bernard, G. Delaizir, J.-C. Sangleboeuf, V. Keryvin, P. Lucas, B.Bureau, X.-H. Zhang, and T. Rouxel, J. Europ. Cer. Soc. 27, 3253 (2007).

${ }^{23}$ J. R. Cost, J. Appl. Phys. 54, 2137 (1983).

${ }^{24}$ R. Fourer, D. M. Gay, and B. W. Kernighan, AMPL: A Modeling Language for Mathematical Programming (Scientific, South San Francisco, CA, 1993).

${ }^{25}$ A. Wächter and L. T. Biegler, Mathematical Programming 106(1), 25 (2006).
${ }^{26}$ R. Kohlrausch, Ann. Phys. Leipzig 91, 56 (1854).

${ }^{27}$ A. S. Nowick and B. S. Berry, Anelastic Relaxation in Crystalline solids (Academic, New York 1972).

${ }^{28}$ R. S. Lakes, Viscoelastic Solids (CRC, Boca Baton, FL, 1999).

${ }^{29}$ H. Kato, H. Igarashi, and A. Inoue, Mater. Lett. 62, 1592 (2008).

${ }^{30} \mathrm{~F}$. Spaepen, private communication.

${ }^{31}$ D. Pan, A. Inoue, T. Sakurai, and M. W. Chen, Proc. Nat. Acad. Sci., U.S.A. 105, 14769 (2008).

${ }^{32}$ D. Pan, Y. Yokoyama, T. Fujita, Y. H. Liu, S. Kohara, A. Inoue, and M. W. Chen, J. Appl. Phys. 95, 141909 (2009).

${ }^{33}$ C. A. Schuh, T. G. Nieh, and Y. Kawamura, J. Mater. Res. 17, 1651 (2002).

${ }^{34}$ W. H. Jiang and M. Atzmon, J. Mater. Res. 18, 755 (2003).

${ }^{35}$ A. Concustell, M. A. Carpenter, and A. L. Greer, private communication.

${ }^{36} \mathrm{H}$. Ullmaier and W. Schilling, in Radiation Damage in Metallic Reactor Materials (IAEA, Vienna, 1980).

${ }^{37}$ P. M. Bronsveld, J. Groote Schaarsberg, J. Nauta, and J. Th. M. De Hosson, Mater. Sci. Eng. 97, 541 (1988).

${ }^{38}$ A. Kursumovic and B. Cantor, Scr. Mater. 34, 1655 (1996).

${ }^{39}$ K. Csach, V. Ocelik, J. Miskuf, V. Bengus, and P. Duhaj, Trans. IEEE 30, 496 (1994), and references therein.

${ }^{40}$ A. Kursumovic and B. Cantor, Scr. Mater. 34, 1655 (1996).

${ }^{41}$ K. Schröter, G. Wilde, R. Willnecker, M. Weiss, K. Samwer, and E. Donth, Eur. Phys. J. B 45, 1 (1998).

${ }^{42}$ Y. Hiki, Mater. Sci. Eng. A 370, 253 (2004).

${ }^{43}$ H. T. Jeong, E. Fleury, W. T. Kim, D. H. Kim, and K. Hono, J. Phys. Soc. Jap. 73, 3192 (2004).

${ }^{44}$ J. M. Pelletier, J. Alloys Comp. 393, 223 (2005)

${ }^{45}$ L. M. Wang, R. Liu, and W. H. Wang, J. Chem. Phys. 128, 164503 (2008).

${ }^{46}$ D. Rodney and C. A. Schuh, Phys. Rev. Lett. 102, 235503 (2009). 